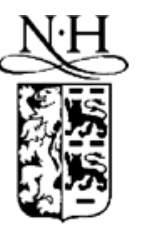

\title{
Status of ANTARES in Nov 2005
}

\author{
J.Carr on behalf of ANTARES Collaboration \\ Centre de Physique de Particules de Marseille, 13288 Marseille, France
}

Elsevier use only: Received date here; revised date here; accepted date here

\begin{abstract}
This article reports on the status of the ANTARES Neutrino Telescope project as of November 2005. The highlights of the activities during 2005 are the deployment and operation of an instrumentation line which has been taking data since April 2005 and the deployment of a complete mechanical test line in the spring of 2005. The first data from the operation of the instrumentation line is presented. The status report gives the plans for the construction of the full ANTARES detector which is planned for completion in 2007.
\end{abstract}

(C) 2001 Elsevier Science. All rights reserved

Keywords : Neutrino astronomy; ANTARES; neutrino telescope; deep sea technology

\section{Introduction}

In 2005 major advances have been made in the ANTARES project with the deployment and operation of two lines: "MILOM" and "LINE0". The MILOM line is a version of the instrumentation line planned in the Technical Design Report [1] with the addition of optical to enable an evaluation in the sea all aspects of the ANTARES detector. The LINE0 operation was included in the program as a necessary step to qualify certain aspects of the line design before entering full production. In particular this line was aimed to test the line cables and the water tightness of all the line elements. These lines, deployed and operated in 2005, follow earlier test lines deployed and operated in 1999/2000 and 2002/2003.

In parallel with the operation of these latter two development lines, the production of most parts of the full ANTARES detector is underway and the integration of the first complete detector line is in progress.

\section{Early Test Lines}

Before the deployment of the MILOM and LINE0, the ANTARES program made use of other test lines connected to the shore via undersea cables. The first such line, referred to as the "Demonstrator Line”, was deployed in November 1999 and connected on the deployment boat to an old undersea cable going to Marseille. This line was operated until 
June 2000 and proved various concepts of the ANTARES design, in particular the acoustic positioning system, and included reconstruction of cosmic ray muons with 7 optical modules [3] as shown in figure 1 . This line used an already existing undersea cable donated by France Telecom to take

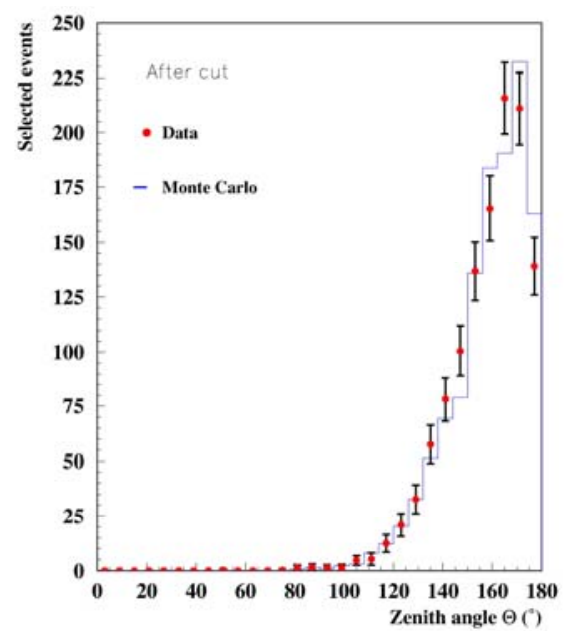

Figure 1. Reconstruction of cosmic ray muons with 1999/2000 Demonstrator Line. The plot corresponds to a total of $\sim 70$ hours of data taking.

data in a telephone exchange centre in Marseille with the demonstrator line being located on a special site near Marseille. In 2001 a new cable was deployed between the final ANTARES site and the shore station in La Seyne-sur-Mer and in 2002 the detector junction box was deployed and connected at the end of this cable. The next test lines were deployed and connected between November 2002 and March 2003. Two lines were deployed, the "MIL", the first version of the instrumentation line and the "PSL", a short prototype for a full optical detector line with 15 optical modules. These lines which were operated in situ until May/ July 2003, respectively, again proved various aspects of the design but indicated certain problems with loss of optical transmission in the line electro-mechanical cables (EMC) and leaks in the cables and containers. The PSL was able to measure the counting rates in the optical modules, in particular the rates of bioluminescence over a period of $\sim 4$ months.

After the experiences of the MIL and PSL lines significant changes were made to the detector design and many quality control procedures were introduced. The MILOM and the LINE0 were chosen as the next steps in the development phase of the project.

\section{Operation of MEOC and Junction Box}

The Main Electro-Optical Cable (MEOC) and Junction Box (JB) have now been in operation for $2 \frac{1}{2}$ years. The MEOC was deployed in the sea in Nov. 2001, initially with only a loop-back container at the sea end. In Nov. 2002 this sea end of the cable was brought to the surface, connected to the junction box and redeployed. Since this date the battery operated slow control in the junction box has sent to shore measurements of various parameters in the JB. Details of this aspect of the ANTARES detector can be found in reference [2].

\section{MILOM Line}

The MILOM line is illustrated in figure 2. The MILOM was deployed in the sea from the ship CASTOR on 18 March 2005 and connected with the Remote Operated Vehicle VICTOR on 12 April 2005.

The MILOM line is equipped with the latest electronics card versions and enables a complete test of every aspect of the detector design. For the final validation of the design, it is the operation of the optical modules, the optical calibration systems and the acoustic positioning systems which are of primary importance. The other instruments of the line are for special investigations and long term monitoring.

It is planned that there could be a continual evolution of the items contained on the instrumentation lines. Some of the instruments require maintenance and some others are developments which could require modifications. Further it is expected other instruments could be added as need or interest arises and for this reason it is expected that the instrumentation lines, including the MILOM could be recovered from the sea more frequently than the standard optical detector lines. 


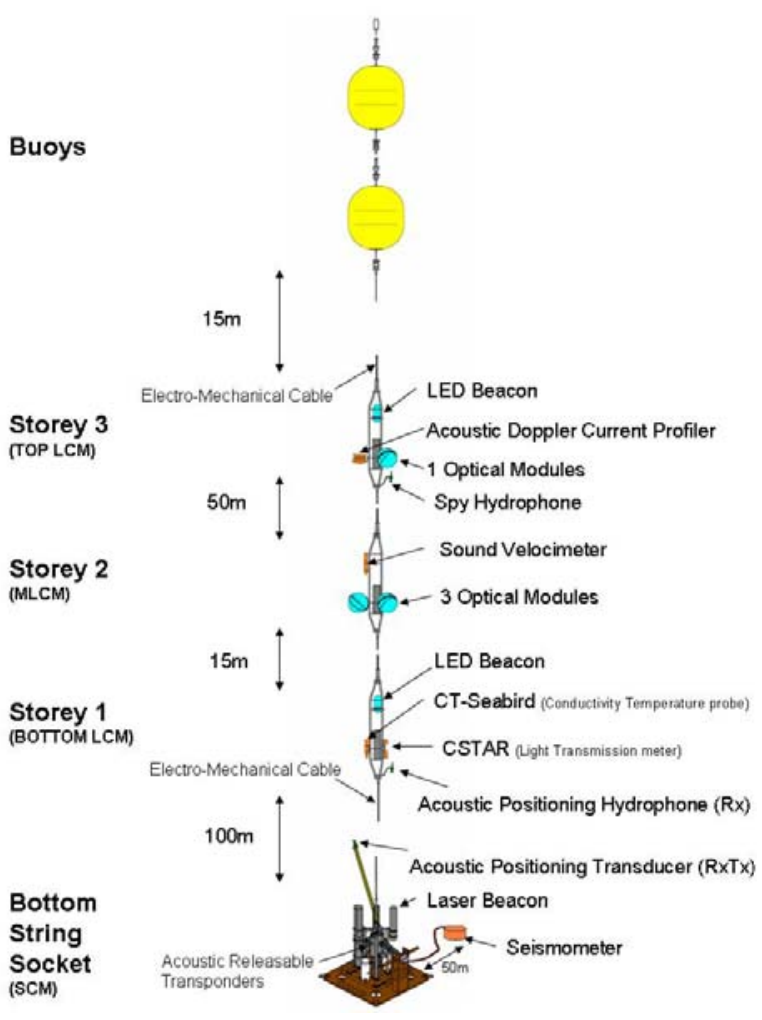

Figure 2. Layout of MILOM line

\section{Results from MILOM operation}

Data taking started with the MILOM immediately after the connection on 12 April. The MILOM operation has provided the opportunity to test and develop all aspects of the data acquisition and analysis software. With the MILOM the full precision of the optical module system has been demonstrated in contrast with the PSL where it was only possible to record the raw counting rates.

\subsection{Optical Module Timing Precision}

The excellent angular resolution of the ANTARES neutrino telescope $\left(<0.3^{\circ}\right.$ for $\mathrm{E}>10$ $\mathrm{TeV})$ relies on good timing resolution of the light signals recorded in the optical modules. The specification for the detector is that the timing resolution is limited by the transit time spread of the photomultipliers which have $\sigma \sim 1.3$ ns. To achieve this all electronics and calibration systems are each required to contribute $<0.5 \mathrm{~ns}$ to the overall timing resolution.

To obtain this necessary resolution in the electronics, a very precise time reference clock distribution system has been implemented. Although this system has been extensively proven in the laboratory it has not been previously possible to demonstrate the same resolution in the sea. In the operation of the PSL this was due to the transmission problem on the optical fibre used by the line clock system.

With the operation of the MILOM the clock has now been fully proven in the sea environment. Figure 3 shows the variation in the measured delay in the clock system within the MILOM between the SCM and the top storey, indicating a time resolution $\sim 0.01 \mathrm{~ns}$ which is very much within the specification.

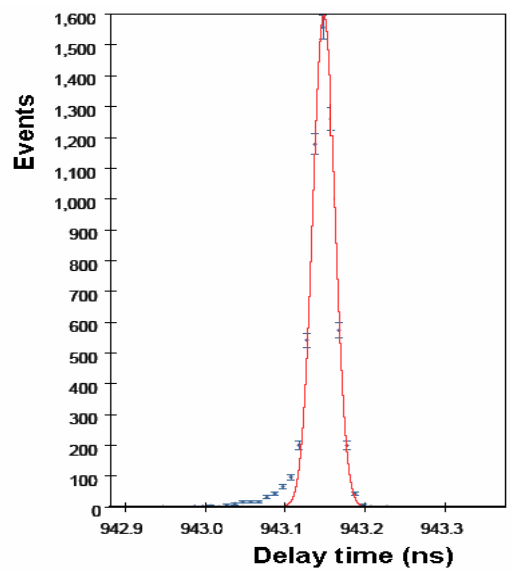

Figure 3. Spread in the time delay as measured in the clock reference system between the SCM and TOP LCM.

The complete timing resolution of the optical modules has been measured with the MILOM using the optical beacon system. As indicated in figures 2, the LED beacon is located in the first storey and the three optical modules in the second storey at a distance of about $15 \mathrm{~m}$. The LED beacon contains 36 individual LEDs synchronised in time and arranged to give a reasonably isotropic light emission. A small PMT internal to the LED beacon monitors the output light pulse time spread and amplitude. 
The test on the timing resolution in the optical modules is performed by pulsing the LED beacon with a frequency of a few Hz. For events where two or more optical modules in the same storey record signals within a certain time window, figure 4 shows the difference between the signals of two adjacent OMs. The timing resolution of one optical module can be estimated to be $\sim 0.5 \mathrm{~ns}$ since two OMs contribute such that the width is $\sqrt{2} \times 0.5=$ $0.75 n s$. This resolution is for large light pulses and so is not dominated by the PMT transit time spread but by the intrinsic electronics resolution. This result shows that the complete electronics contribution is $<0.5$ ns.

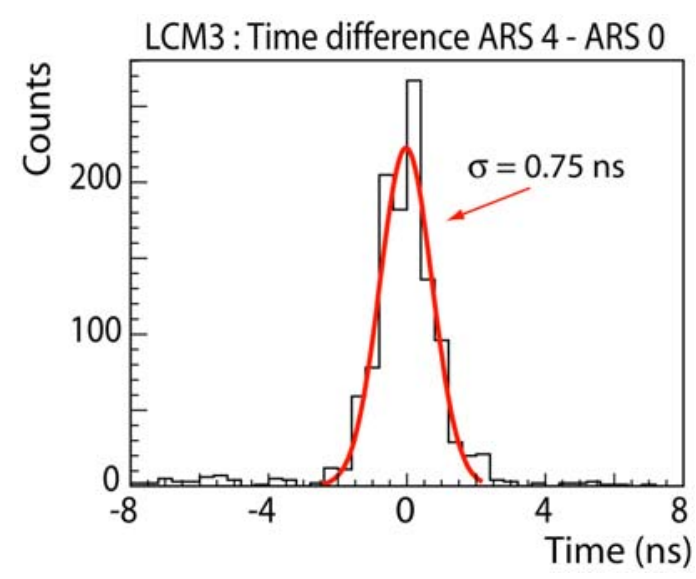

Figure 4. An example of the distribution of difference in time between signals between adjacent optical modules in the storey.

Each optical module contains an internal LED, mounted on the back of the photomultiplier, in order to monitor the stability of the photomultiplier. Figure 5 shows the data from this system in for two optical modules on various days, both before deployment in the laboratory and in situ in the sea. It can be seen that a shift of $\sim 3 n s$ takes place between the shore and sea data, most likely due to temperature effects in the LED pulsing circuit and that the OMs are stable to $<1$ ns during operation in the sea.

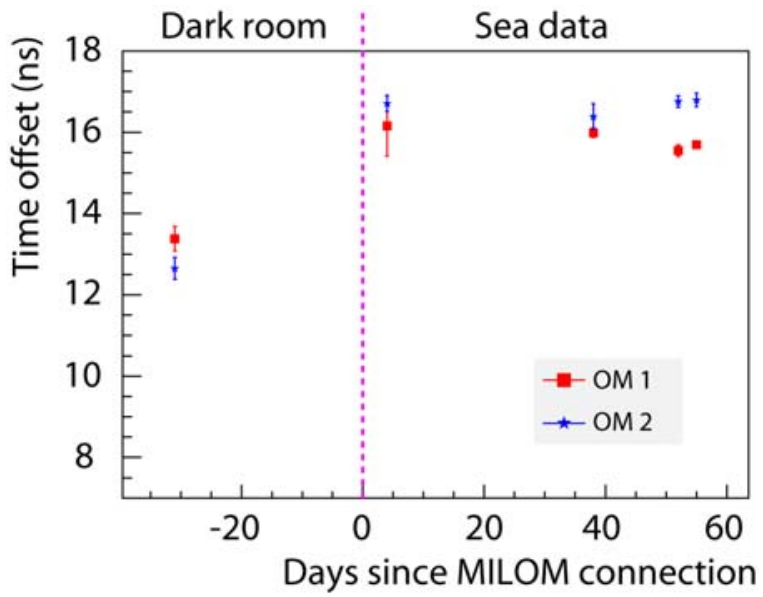

Figure 5. Data from the OM internal LED system.

\subsection{Acoustic Positioning System Resolution}

The second essential element to realise the necessary angular resolution of the neutrino telescope, is the knowledge of the position in 3D space of the optical modules with a precision $\sim 10 \mathrm{~cm}$ in real time. These position measurements are provided by the acoustic positioning system. As mentioned above, the demonstrator line showed the required precision from a prototype of this system; however the PSL/MIL line were not able to provide precise data because of the fibre optics faults in the clock. As for the timing precision, the MILOM has provided the final proof that ANTARES can achieve the necessary spatial position resolution and so complete the confidence that the good angular resolution will be obtained in the full ANTARES detector.

The full acoustic positioning system will consist of a three dimensional array of transponders and receivers, on or near the sea bed, exchanging precisely timed acoustic signals between each other. At the present time only a limited number of devices are installed on the site. Figure 6 shows the precision of the measurement between two fixed points on the MILOM line to be $0.2 \mathrm{~cm}$ for the measurement of a distance $\sim 100 \mathrm{~m}$. 


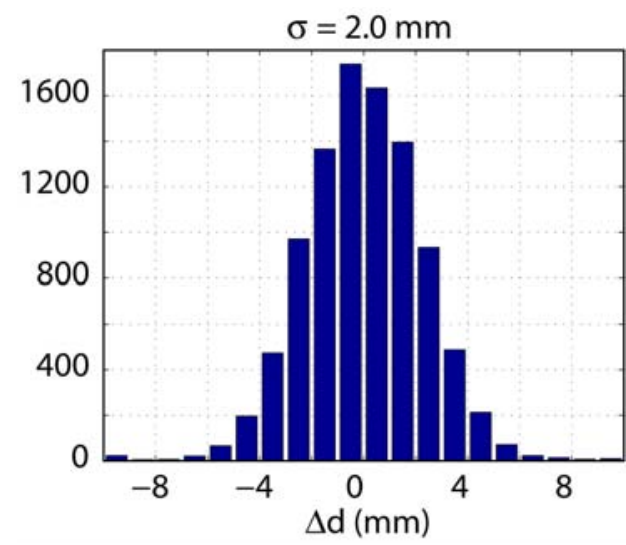

Figure 6 . The variation on acoustic distance measurement between the two fixed points in the MILOM. Here an average position is calculated for each day and the difference is taken to this average.

These first available data from the MILOM indicate that all features of the acoustic positioning system are functional on the sea bed and that their precision is well within the required specifications. A conclusion on the ultimate positioning precision must await a complete analysis, including all corrections; however it seems already that the specification of $<10 \mathrm{~cm}$ spatial precision will be possible.

\subsection{Environment Instruments}

The Acoustic Doppler Current Profiler (ADCP) on the MILOM measures the sea current as a function of depth from the top storey on the MILOM down $160 \mathrm{~m}$ to the sea bed. Figure 7 shows an example of the data corresponding to a depth close to the top of the line. Another important instrument on the MILOM is the CSTAR device which measures the water transparency. Although the data quality from this instrument appears to be good, the calibration is not yet understood. The CT-Seabird device measures the sea temperature and conductivity from which the salinity and density can be calculated. A separate instrument measures the sound velocity. Each storey of the MILOM contains a compass/tiltmeter to measure the line orientation. These devices are standard throughout the ANTARES detector and supplement the position information from the acoustic positioning devices, both adding precision and providing checks. Figure 8 shows the compass heading for the three MILOM storeys.

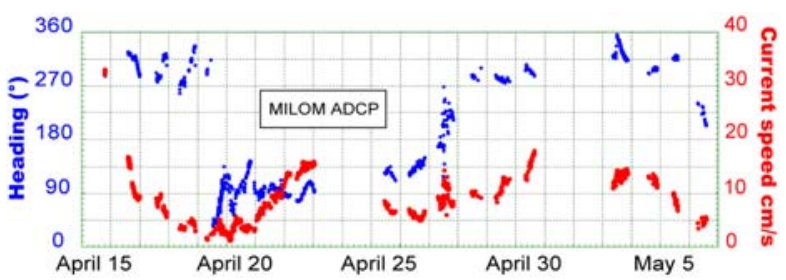

Figure 7. Data from the ADCP measuring sea current speed and direction from 15 Apr to 7 May.

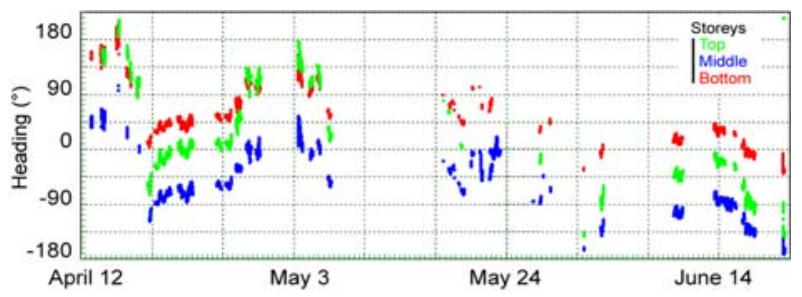

Figure 8. Heading data from compasses in each MILOM storey.

\section{LINE 0}

LINE 0 is the first mechanically complete line deployed by ANTARES. It contains 23 storeys and is very close in all mechanical aspects to a final line, but it contains none of the electronics being tested in the MILOM for the full line production. Various autonomous recording devices enabled monitoring of fibre optic transmission, leaks and aspects of electrical connections The LINE 0 deployment took place in March and was connected to the junction box with VICTOR in April. After two months in the sea with one month being connected to the shore the LINE 0 was recovered in May.

After recovery the situation was diagnosed. There were no water leaks in electronics containers and no electrical conductivity or insulation problems; there were however optical transmission losses at a number of locations in the line. The disassembly of the line has lead to an initial understanding of the cause of the optical transmission losses. All optical fibre losses are inside the electronics containers and not inside the EMC cables themselves. Hence the 
present problem is different in nature from that observed in the PSL/MIL where the problem was due to optical fibre breaks and sheathing contact with the cable itself.

The new problem arises at the interface between the EMC penetrator and the LCM cylinder. It is observed that the core of the EMC cable is not sufficiently well fixed inside the penetrators and moves inside the LCM cylinders. From the observations of the disassembled LINE 0 , it seems that the maximum displacement of a cable core through the penetrators was $\sim 30 \mathrm{~mm}$. With this amount of movement the fibres entering the cylinder were pushed against a fixed object, in some cases breaking fibres and in other cases causing tight bends and attenuation. In total about half of the cable penetrators in the line had some movement, of those which moved around half moved $\sim 30 \mathrm{~mm}$ and the rest $5-20 \mathrm{~mm}$. A solution to this electro-mechanical cable penetrator problem is being developed with the manufacturer.

\section{Full detector production status}

During 2003 and 2004, the various qualifications and tests in the sea led step by step to decisions to launch the orders for the full quantity of nearly all elements for the 12 line ANTARES detector. The only items not so ordered are the EMC cables where only elements for two lines and the MILOM have been ordered. In addition, a few orders have been split in parts to correspond to the available funding.

Given these decisions and orders, at the present time all detector components are available for the production of the first complete detector line, LINE 1. For many components the items are available for many lines, in some cases for all lines and by the autumn of 2005 nearly all items will be available for the first six lines of the detector. Again, the exception will be the EMC cables. The assembly of the LINE 1 is expected to be complete in December.

The operation of the MILOM and LINE 0 has been an immense step forward in the ANTARES project. The elements necessary for obtaining the excellent angular resolution of the detector have been proven. The improvements resulting from the changes made following the PSL and MIL operations have essentially all been verified. The solution to the new optical transmission problem is being developed. A very favourable result of the MILOM and LINE 0 deployments is the total absence of water leaks in the containers and cables in distinction to the situation with the PSL and MIL.

The successful deployment of the MILOM and LINE 0, have again proven the facility of the deployment and connection methods. The initial data recorded with the MILOM already demonstrate that the specifications of timing resolutions $<0.5 \mathrm{~ns}$ in the electronics and calibration systems can be achieved. The analysis of the acoustic positioning data is not complete but all indications are that here also the design resolution will be achieved. All the environment instruments on the MILOM are operational with extensive data collected. At the present time of June 202005 the MILOM is fully operational and data taking will continue for several months. These data will give further vital information on the stability of the full electronics system and the quality of the data.

The final line assembly of the series production of the 12 ANTARES lines will rapidly start $^{1}$. In the peak of the production process it is planned to deploy approximately one line per month during 2006 and with this schedule, it is planned that the detector will be complete in 2007 .

\section{References}

[1] Technical Design Report: http://antares.in2p3.fr/internal/tdr/contents.html

[2] G Hallewell, these proceedings.

[3] A. Kouchner, thesis: http://antares.in2p3.fr/Publications/thesis/2001/an toine-kouchner-phd.ps.gz

\footnotetext{
${ }^{1}$ In early 2006 the LINE 1 was successfully deployed and connected.
} 
